Hirschel Valiente Rouco

Influência da Distribuição Espacial do Reforço nas Propriedades de Compósitos: Simulação e Caracterização de Microestruturas. Modelagem por Elementos Finitos

Tese de Doutorado

Tese apresentada como requisito parcial para obtenção do título de Doutor pelo Programa de PósGraduação em Engenharia de Materiais e de Processos Químicos e Metalúrgicos do Departamento de Engenharia de Materiais da PUC-Rio.

Orientador: Prof. Sidnei Paciornik Co-Orientador: Prof. José Roberto Moraes d’Almeida 
Hirschel Valiente Rouco

\section{Influência da Distribuição Espacial do Reforço nas Propriedades de Compósitos: Simulação e Caracterização de Microestruturas. Modelagem por Elementos Finitos}

Tese apresentada como requisito parcial para obtenção do título de Doutor pelo Programa de Pós-Graduação em Engenharia de Materiais e de Processos Químicos e Metalúrgicos da PUC-Rio. Aprovada pela Comissão Examinadora abaixo assinada.

Prof. Sidnei Paciornik

Orientador

Departamento de Engenharia de Materiais - PUC-Rio

Prof. José Roberto Moraes d'Almeida

Co-Orientador

Departamento de Engenharia de Materiais - PUC-Rio

Profa. Lavinia Maria Sanabio Alves Borges

UFRJ

Prof. Norberto Cella

UERJ

Prof. Roberto Ribeiro de Avillez

Departamento de Engenharia de Materiais - PUC-Rio

Prof. Marcos Henrique de Pinho Mauricio

Departamento de Engenharia de Materiais - PUC-Rio

Prof. José Eugênio Leal

Coordenador Setorial do Centro Técnico Científico - PUC-Rio 
Todos os direitos reservados. É proibida a reprodução total ou parcial do trabalho sem autorização da universidade, da autora e do orientador.

\section{Hirschel Valiente Rouco}

Graduado em Física pela Universidade de Havana. Mestre em Física pela Universidade de Havana.

Ficha Catalográfica

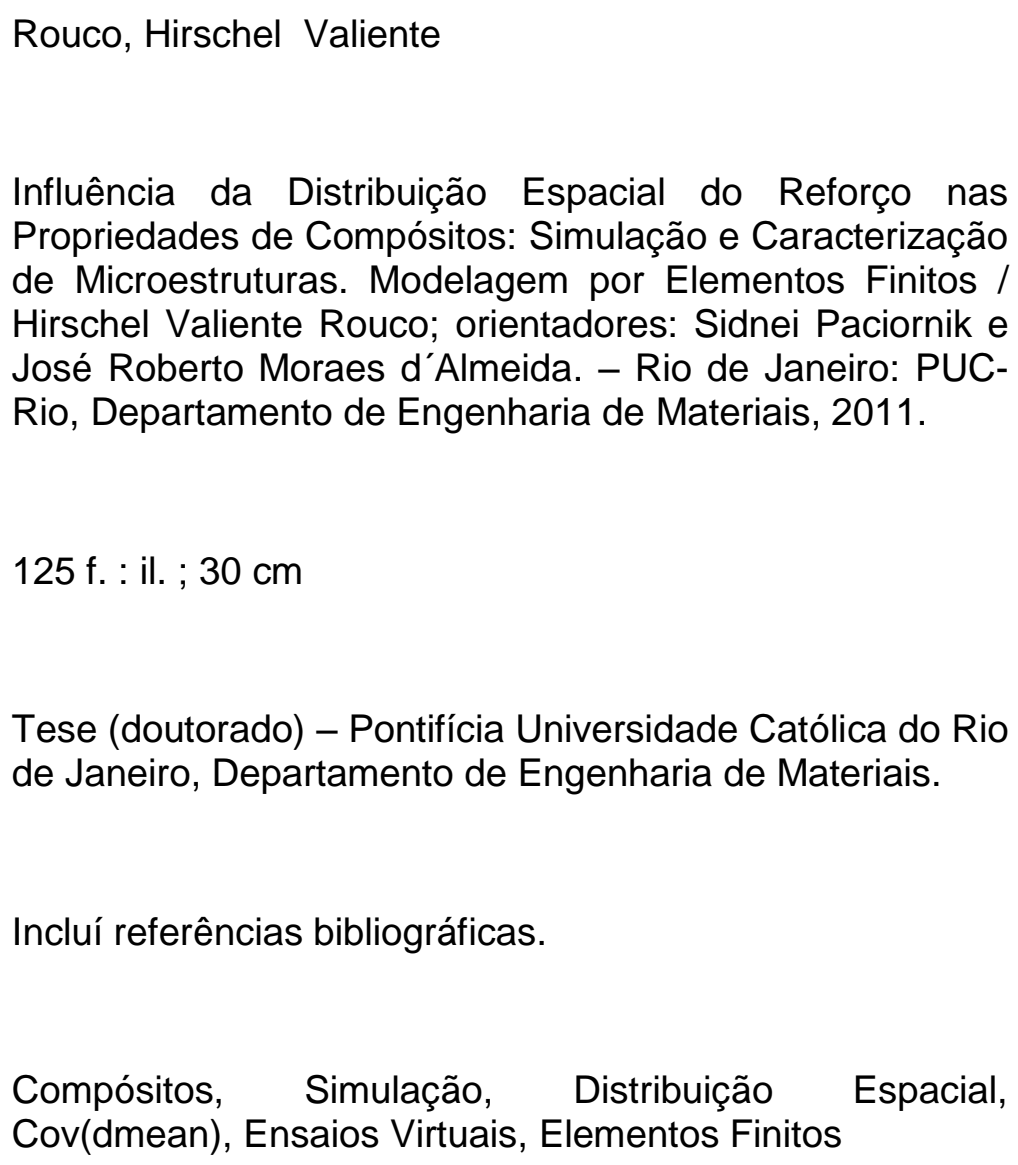
de Microestruturas. Modelagem por Elementos Finitos / Hirschel Valiente Rouco; orientadores: Sidnei Paciornik e José Roberto Moraes d'Almeida. - Rio de Janeiro: PUCRio, Departamento de Engenharia de Materiais, 2011.

125 f. : il. ; $30 \mathrm{~cm}$

Tese (doutorado) - Pontifícia Universidade Católica do Rio de Janeiro, Departamento de Engenharia de Materiais.

Incluí referências bibliográficas.

Compósitos, Simulação, Distribuição Espacial, Cov(dmean), Ensaios Virtuais, Elementos Finitos 


\section{Agradecimentos}

Agradeço aos meus dois orientadores doutores Sidnei Paciornik e José Roberto d’Almeida pela orientação e a ajuda recebida, e por manter a fé em mim.

Agradeço ao Brasil e ao $\mathrm{CNPq}$ por fazer possível minha formação de doutor. 


\section{Resumo}

Rouco, Hirschel Valiente; Paciornik, Sidnei; d’Almeida, José Roberto Moraes. Influência da Distribuição Espacial do Reforço nas Propriedades de Compósitos: Simulação e caracterização de Microestruturas. Modelagem por Elementos Finitos. Rio de Janeiro, 2011. 125p. Tese de Doutorado Departamento de Engenharia de Materiais, Pontifícia Universidade Católica do Rio de Janeiro.

É um fato conhecido que o nível de dispersão da carga (reforço) num compósito assim como as características da sua distribuição espacial tem uma estreita relação com as propriedades do material. Neste sentido, a caracterização e o controle da distribuição espacial e/ou dispersão da carga no volume da matriz em materiais compósitos é um problema importante no qual atualmente se trabalha em várias frentes. O presente trabalho aprofunda no uso do coeficiente de variação da distância média aos vizinhos mais próximos $\operatorname{Cov}($ dmean) como parâmetro de caracterização da distribuição da carga em compósitos. São pesquisados os limites de uso deste parâmetro para a identificação de distribuições randômicas e homogêneas de partículas em imagens 2D. Para realizar este estudo foi necessário desenvolver diferentes códigos de programação com o fim de simular imagens de configurações espaciais características de corpos (distribuição randômica e homogênea, presença de clusters, zonas de exclusão), e caracterizá-las. Como resultado desta pesquisa é expandida a área de aplicação da metodologia que usa o Cov(dmean) para estudar a presença de distribuições randômicas e homogêneas em imagens com concentrações menores do que 25\% (em área de corpos). Também é sugerido um limite para o número mínimo de corpos em imagens e campos locais, abaixo do qual o critério oferecido pelo valor do $\operatorname{Cov}($ dmean) não é confiável. Uma nova ferramenta computacional é desenvolvida e apresentada: "mapas de Cov(dmean)". Ela permite estudar a presença de distribuições espaciais características em campos locais entre outras potencialidades expostas na tese. Também, como parte deste trabalho de tese, são realizados ensaios virtuais usando o software de elementos finitos OOF2, para determinar propriedades em materiais compósitos reais e virtuais com vários tipos de distribuição espacial do reforço, observando a influência da distribuição da carga nas propriedades finais do compósito.

\section{Palavras-chave}

Compósitos; Simulações; Distribuição Espacial; Cov(dmean); Elementos Finitos 


\section{Abstract}

Rouco, Hirschel Valiente; Paciornik, Sidnei (Advisor); d’Almeida, José Roberto Moraes (Co-Advisor). Influence of the reinforcement spatial distribution on the properties of composites. Simulation and Characterization of Microstructures. Modeling by Finite Elements. Rio de Janeiro, 2011. 125p. Doctoral Thesis - Departamento de Engenharia de Materiais, Pontifícia Universidade Católica do Rio de Janeiro.

It is a known fact that the level of dispersion of the load (reinforcement) in a composite and the characteristics of its spatial distribution has a close relationship to the material properties. In this sense, the characterization and control of the spatial distribution and / or spread of the load in the volume of the matrix in composite materials is an important problem that is currently under research on several fronts. This thesis promotes the use of the coefficient of variation of the average distance to nearest neighbors $\operatorname{Cov}($ dmean) as a parameter characterizing the load distribution in composites. In this work, the limits of use of this parameter for the identification of random and homogeneous distributions of particles in 2D images are investigated. To conduct this study it was necessary to develop different programming codes in order to simulate images of characteristic spatial configurations of bodies (random, homogeneous distribution, presence of clusters, zones of exclusion), and characterize them. As a result of this research the area of application of the methodology that uses the Cov (dmean) to study the presence of random and homogeneous distributions in images is expanded to lower concentrations below 25\% (in area of bodies). Moreover a limit to the minimum number of bodies in images and local fields is suggested, below which the criteria offered by the value of $\operatorname{Cov}(d m e a n)$ is unreliable. A new computer tool

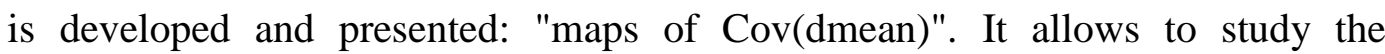
presence of spatial distributions in local fields and other application presented in the thesis. Also, as part of this thesis, virtual tests are conducted using the finite element software OOF2 to determine properties of real and virtual composites with various types of spatial distributions of reinforcement, observing the influence of the load distribution on the final properties of the composite.

\section{Keywords}

Composites; Simulations; Spatial Distribution; Cov(dmean); Finite Elements 


\section{Sumário}

1 Introdução 15

2 Revisão Bibliográfica

2.1. Materiais compósitos, classificações. Distribuição espacial da carga e propriedades.

2.2. Definição de ensemble espacial de partículas. Ensemble real e ensemble virtual.

2.3. Ensemble homogêneo randômico (random homogeneous ensemble RHE)

2.3.1. Técnicas para simular RHE. Ensemble por sequiência de adição randômica (random sequential addition ensemble RSAE)

2.4. Caracterização da distribuição espacial de corpos num ensemble. Parâmetros espaciais.

2.4.1. Medidas de frequência espacial $\quad 24$

2.4.2. Operação de "tessellation" e parâmetros espaciais derivados.

2.5. O coeficiente de variação da distancia media aos vizinhos mais próximos (Covdmean) como identificador de um ensemble homogêneo randômico RHE.

2.6. Softwares de elementos finitos. OOF2: Testes virtuais para a estimação de propriedades de materiais compósitos 
3 Algoritmos e metodologia $\quad 37$

3.1. Construção de ensembles virtuais 37

3.1.1. Determinação das coordenadas de cada partícula num RHE 39

3.1.2. Determinação das coordenadas de cada partícula em ensembles com distribuição espacial heterogênea (clusters) 40

3.1.3. Determinação das coordenadas de cada partícula em ensembles com distribuição espacial heterogênea (áreas de exclusão) 42

3.2. Determinação do $\operatorname{Cov}_{\text {dmean }}$ de ensembles reais e virtuais (imagens 2D)

3.3. Cálculo do $\operatorname{Cov}_{\text {dmean }}$ local e geração de mapas de $\operatorname{Cov}_{\text {dmean }}$

3.4. Metodologia 49

3.4.1. Corroborando os resultados de N. Yang e co-autores [33] 49

3.4.2. Estendendo a validade dos resultados de N. Yang e co-autores [33] 52

3.4.3. Construindo mapas de $\operatorname{Cov}_{\text {dmean }}$

3.5. Algoritmo e metodologia para a implementação de ensaios virtuais. $\quad 55$

3.5.1. Materiais $\quad 55$

3.5.2. Definição do problema físico $\quad 57$

3.5.3. Aplicação do método de elementos finitos 58

$\begin{array}{ll}4 \text { Resultados e discussão } & 61\end{array}$

4.1. Verificação dos resultados de N. Yang e co-autores [33] 61

4.2. Ampliando a área de validade dos resultados de

N. Yang e co-autores [33] 63 
4.3. Geração de mapas de $\operatorname{Cov}_{\text {dmean }}$. Potencialidade e aplicações

4.4. Implementação de ensaios virtuais. Resultados

69

5 Conclusões e Recomendações para Trabalhos Futuros

5.1. Conclusões 74

5.2. Recomendações

6 Referências

7 Apêndice 1 - Códigos computacionais para a construção de ensembles virtuais

8 Apêndice 2 - Código computacional para a determinação do Covdmean de ensembles reais e virtuais.

9 Apêndice 3 - Códigos computacionais para a determinação do Covdmean local e para a construção de mapas de Covdmean 


\section{Lista de figuras}

Figura 1 - Esquema de relação entre os parâmetros de distribuição espacial, as variáveis de processamento e as propriedades do material.

Figura 2 - Classificação dos materiais compósitos. Imagens dos três casos de compósitos reforçados por fibras

Figura 3 - Idéia geral do algoritmo RSAE: quando a coordenada randômica da partícula implica superposição com uma partícula que já existe, esta é gerada outra vez (partícula 3).

Figura 4 - Medidas básicas de frequiência espacial na direção do eixo "x". a) Contagem no segmento, b) Contagem nas áreas

Figura 5 - a) "tessellation" de Dirichlet aplicada a um ensemble espacial de corpos circulares idênticos. b) "tessellation" de Voronoi aplicada a um ensemble espacial de corpos com distribuição de tamanho, forma e orientação heterogênea.

Figura 6 - "tessellation" de Voronoi: As fronteiras da célula do corpo definem os vizinhos mais próximos.

Figura 7 - Ensembles de partículas com distribuição espacial homogênea. a) Forma circular, distribuição de tamanho uniforme. b) Forma circular, distribuição de tamanho normal. c) Forma circular distribuição de tamanho exponencial. d) Forma elipsoidal, distribuição de tamanho uniforme, distribuição de orientação uniforme. e) Forma elipsoidal, distribuição de tamanho uniforme, distribuição de orientação randômica. f) Parâmetros de forma randômicos, distribuição de tamanho não uniforme, distribuição de orientação randômica.

Figura 8 - Ensembles de partículas com distribuição espacial heterogênea. a) Áreas de exclusão isotrópicas. b) Clusters isotrópicos. c) 
Figura 9 - Resultados do trabalho de Yang e co-autores [33]: Coeficiente de variação de parámetros de "tessellation" de Voronoi para distribuições simuladas homogêneas e heterogénea (zonas de exclução).

Figura 10 - Evolução do $\operatorname{Cov}_{\text {dmean }}$ com a diminuição do tamanho de partículas (878 partículas)

Figura 11 - Área de validade dos resultados de Yang e co-autores (2001) [33]

Figura 12 - Interface inicial do OOF2

Figura 13 - Esquema geral do OOF2

Figura 14 - Esqueleto associado à imagem transversal de um compósito virtual de fibras infinitas e paralelas. (branco: fibra, azul: matriz).

Figura 15 - Estrutura macroscópica dos algoritmos usados

Figura 16 - Fluxograma do algoritmo genérico para a determinação das coordenadas de cada partícula num RHE. A descrição dos elementos A-F é fornecida no texto do corpo da tese.

Figura 17 - Fluxograma do algoritmo genérico para a determinação das coordenadas de cada partícula em ensembles com distribuição espacial heterogênea formando clusters. A descrição dos elementos A - I é fornecida no texto do corpo da tese.

Figura 18 - Fluxograma do algoritmo genérico para a determinação das coordenadas de cada partícula em ensembles com distribuição espacial heterogênea com zonas de exclusão. A descrição dos elementos A - H é fornecida no texto do corpo da tese.

Figura 19 - Esquema geral do algoritmo para a obtenção do $\operatorname{Cov}_{\text {dmean }}$

Figura 20 - Algoritmo básico usado para aplicar a operação de "tessellation" deVoronoi. 
Figura 21 - Exemplo de aplicação da "tessellation” deVoronoi: $\operatorname{Img}_{0}$ (esquerda), $\operatorname{Img}_{\text {Tess }}$ (direita)

Figura 22 - Algoritmo da segunda etapa: determinação do $\operatorname{Cov}_{\text {dmean }}$.

Figura 23 - Rotina para a determinação do $\operatorname{Cov}_{\text {dmean }}$ local.

Figura 24 - Rotina para a construção do mapa de $\operatorname{Cov}_{\text {dmean }}$.

Figura 25 - Algoritmo usado para a construção e caracterização de ensembles

Figura 26 - Área de validade dos resultados Yang e co-autores [33] (verde): Novas direções de pesquisa propostas

Figura 27 - Algoritmo usado para criar e caracterizar ensembles diminuindo o número de partículas sem alterar a fração de área.

Figura 28 - Algoritmo usado para criar e caracterizar ensembles diminuindo o número de corpos com o tamanho do campo e dos corpos fixo.

Figura 29 - Imagem para a qual foi construído o mapa de $\operatorname{Cov}_{\text {dmean }}$

Figura 30 - Ensembles usados no experimento virtual. a-c) Ensembles virtuais d) Ensemble real

Figura 31 - Seção transversal de uma viga de compósito reforçado por fibras, deformada na direção x.

Figura 32 - Medições de $\operatorname{Cov}_{\text {dmean }}$ para deferentes tipos de ensembles (Tabela 1)

Figura 33 - Procedimentos usados para criar ensembles virtuais: a) N. Yang e coatures [33] b) Presente Tese

Figura 34 - Medições de $\operatorname{Cov}_{\text {dmean }}$ em função do tamanho do campo de imagens (RHE) de 878 corpos, 15\% de concentração (acima); e de 1463 corpos, $25 \%$ de concentração (abaixo)

Figura 35 - Medições de $\operatorname{Cov}_{\mathrm{dmean}}$ em função do número de corpos 
(direita) e da concentração de corpos (esquerda) em imagens (RHE) de tamanho constante

Figura 36 - Resultados estendidos de N. Yang e co-autores

Figura 37 - Resultado dos testes virtuais nas peças virtuais

Figura 38 - Mapas de tensões da seção transversal das peças 1,2 e 3

deformadas. Imagens a), b) e c) respectivamente. (Escala de cores em $\mathrm{GPa})$. 


\section{Lista de tabelas}

Tabela 1 - Configurações dos ensembles virtuais construídos 51

Tabela 2 - Parâmetros usados nos ensaios virtuais $\quad 57$

Tabela 3 - Mapa de Cov dmean associado à imagem da Figura $29 \quad 67$

Tabela 4 - Interpretação do mapa de $\operatorname{Cov}_{\text {dmean }}$ (Tabela 3) 68 